\title{
Effect of Heat Treatment on the ac Conductivity and Dielectric Properties of $\mathrm{Ag}_{33} \mathrm{Sb}_{31} \mathrm{Se}_{36}$ Thin Films
}

\author{
E. ABD El-WahabB
}

Physics Department, Faculty of Education, Ain Shams University

Roxy, Cairo, Egypt

(Received June 11, 2004; revised version February 21, 2005;

in final form June 21, 2005)

\begin{abstract}
The effect of heat treatment on the ac conductivity and dielectric properties of the melt quenched and thermally evaporated $\mathrm{Ag}_{33} \mathrm{Sb}_{31} \mathrm{Se}_{36}$ chalcogenide system are reported for the first time. The results of the alternating current conductivity $\sigma_{\mathrm{ac}}$, the dielectric constant $\varepsilon_{1}$, and the dielectric loss $\varepsilon_{2}$ of the $\mathrm{Ag}_{33} \mathrm{Sb}_{31} \mathrm{Se}_{36}$ thin film samples are presented over the temperature range $303-373 \mathrm{~K}$ and the frequency range $0.1-100 \mathrm{kHz}$. The temperature dependence of the ac conductivity $\sigma_{\mathrm{ac}}(\omega)$ and the frequency exponent $s$ are discussed with the aim of the correlated barrier hopping model. Values of $\sigma_{\mathrm{ac}}(\omega), \varepsilon_{1}$, and $\varepsilon_{2}$ were found to increase with the increase in the annealing temperature due to the reduction of the number of unsaturated defects which decrease the density of localized states in the band structure.
\end{abstract}

PACS numbers: 73.61.-r, 77.55.+f, 81.40.Rs, 81.40.Tv, 81.40.Ef

\section{Introduction}

The electrical charge transport in the chalcogenide glasses is of considerable interest both from the point of view of basic physics as well as solid state device technology [1]. The common feature of these glasses is the presence of localized states in the mobility gap as a result of the absence of long range order as well as various inherent defects.

Measurements of the frequency and temperature dependence of the ac conductivity and dielectric constants of amorphous chalcogenide semiconductors has been extensively utilized for the understanding of the conduction process in those materials [2].

Studies on semiconducting chalcogenide glasses based on Se have received much attention because of their interesting optical, photoconducting, electrical and magnetic properties. A number of papers [3-6] have appeared in the literature 
reporting various properties of SbSe alloys. $\mathrm{Ag}$ as an additive in chalcogenide glasses (CGs) and particularly thin films of such glasses has attracted widespread interest in glass science [7-10]. The interest stems in part from light-induced effects relevant to optical recording and information processing [6]. It also stems from drastically increased electrical conductivity of CGs [11].

Little attention has been paid to the ternary compound of the type $\mathrm{A}^{\mathrm{I}} \mathrm{B}^{\mathrm{V}} \mathrm{C}^{\mathrm{VI}}$ [12-14] including AgSbSe system. Recently, we have published two papers on the studies of $\mathrm{Ag}_{33} \mathrm{Sb}_{31} \mathrm{Se}_{36}$ system [15, 16]. The first one [15] deals with the effect of thickness on the direct current conductivity $\sigma_{\mathrm{dc}}$, the absorption coefficient $\alpha$, and the optical energy gap $E_{\mathrm{g}}$ of $\mathrm{Ag}_{33} \mathrm{Sb}_{31} \mathrm{Se}_{36}$ as-deposited thin films. The second one [16] deals with the effect of annealing on the optical properties of $\mathrm{Ag}_{33} \mathrm{Sb}_{31} \mathrm{Se}_{36}$ thin films.

The purpose of the present investigation is to obtain more information about the electrical properties of $\mathrm{Ag}_{33} \mathrm{Sb}_{31} \mathrm{Se}_{36}$ thin films. The present study deals with the frequency and temperature dependence of the ac conductivity and the dielectric properties of $\mathrm{Ag}_{33} \mathrm{Sb}_{31} \mathrm{Se}_{36}$ thin films and the effect of heat treatment on it. Thickness and ac conductivity measurements and X-ray diffraction patterns were used.

\section{Experimental details}

Elements of purity $(5 \mathrm{~N})$ were used to obtain the investigated material. They were sealed together in evacuated silica tube under a pressure of $10^{-5}$ Torr. The sealed tube were heated in a suitable furnace where the temperature was raised at a rate of $3-4 \mathrm{~K} \mathrm{~min}^{-1}$ up to $1000 \mathrm{~K}$ and kept around that temperature for $24 \mathrm{~h}$ during which the melt was continuously agitated to achieve homogenization. The tube was finally quenched in granulated ice.

Thin films were prepared by thermal evaporation of the prepared compound from molybdenum boat onto cleaned glass substrates kept at room temperature under a vacuum of $10^{-5}$ Torr using an Edwards E306 A coating unit. Films with different thicknesses in the range $(111.6-180.1 \mathrm{~nm})$ were obtained under a constant rate and the same conditions of deposition.

Multiple-beam Fizeau fringes were used for measuring the film thickness. Energy dispersive X-ray (EDX) spectroscopy analysis was used to determine the composition of the thin film specimens and was found to be the same as that of the starting material. The differential thermal analysis (DTA) of the prepared material was carried out using a Shimadzu model DTA-30 apparatus with a uniform heating rate of $10 \mathrm{~K} \mathrm{~min}^{-1}$. Structural investigation was carried out by using a Philips Pw1373 X-ray diffractometer with $\mathrm{Cu}$ radiation $(\lambda=1.542 \AA)$ and Ni filter operated at $36 \mathrm{kV}$ and $20 \mathrm{~mA}$ with a scanning rate of $2 \mathrm{deg} \mathrm{min}^{-1}$ in the angular range 4 to $90^{\circ}$.

For ac measurements the films were sandwiched between two $\mathrm{Al}$ electrodes. A programmable automatic LCR meter (Philips PM 6304) was used to measure 
directly the impedance $Z$, the capacitance $C$, and the loss tangent $(\tan \delta)$ in the frequency range $0.1-100 \mathrm{kHz}$ and at various temperatures in the range $303-373 \mathrm{~K}$. The total conductivity was calculated from the equation: $\sigma_{\text {tot }}(\omega)=d / Z A$, where $d$ is the film thickness and $A$ is the cross-sectional area. The dielectric constant was calculated from the equation: $\varepsilon_{1}=d C / A \varepsilon_{0}$, where $C$ is the capacitance of the film and $\varepsilon_{0}$ is the permittivity of free space. The dielectric loss $\varepsilon_{2}$ was calculated from the equation: $\varepsilon_{2}=\varepsilon_{1} \tan \delta$ where $\delta=90-\phi$.

\section{Results and discussions}

\subsection{Structure}

The composition of as-prepared thin film samples has been determined using EDX. Figure 1 shows the spectral distribution of the constituent elements which were found to be 33,31 , and $36 \%$, respectively, for $\mathrm{Ag}, \mathrm{Sb}$, and Se. This composition $\mathrm{Ag}_{33} \mathrm{Sb}_{31} \mathrm{Se}_{36}$ is the closest composition that we can get from AgSbSe system.

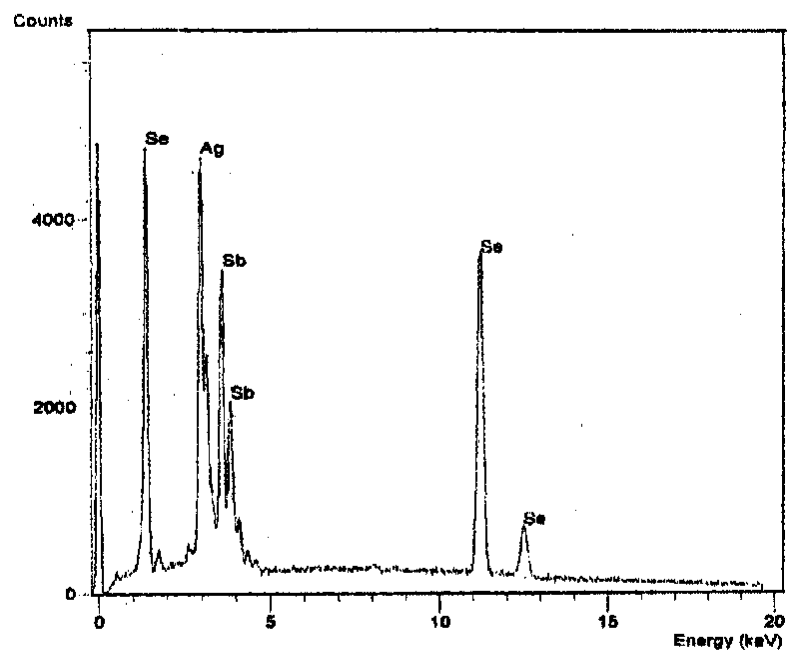

Fig. 1. Energy dispersive X-ray spectrum.

The effect of annealing temperature on the structure of $\mathrm{Ag}_{33} \mathrm{Sb}_{31} \mathrm{Se}_{36}$ thin films is traced through the X-ray diffraction patterns given in Fig. 2 for powder and thin films having the same thickness and deposited at room temperature, then annealed for one hour at different temperatures up to $473 \mathrm{~K}$. This indicated that the powder material has a polycrystalline structure. The as-deposited and annealed films at temperature equal to or less than $423 \mathrm{~K}$ are amorphous. Films that were annealed at $473 \mathrm{~K}$ are partially transformed into a polycrystalline structure characterized by preferred orientation and growth on some crystallographic planes which have lower internal stresses, less orientation energy, and small boundary angles of which the planes (200) and (220) were identified. 


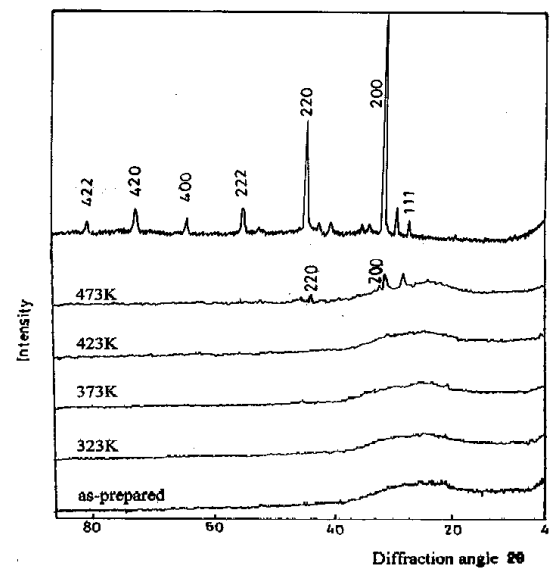

Fig. 2. X-ray diffraction patterns of $\mathrm{Ag}_{33} \mathrm{Sb}_{31} \mathrm{Se}_{36}$ as powder and thin films (thickness $180.07 \mathrm{~nm}$ ) annealed at different temperatures marked on the charts.

\subsection{Frequency and temperature dependence of the ac conductivity}

A feature common to almost all amorphous semiconductors and some other disordered systems is that the ac conductivity $\sigma_{\mathrm{ac}}(\omega)$ increases with increasing frequency according to the power-law relation

$$
\sigma_{\mathrm{ac}}(\omega)=\sigma_{\mathrm{tot}}-\sigma_{\mathrm{dc}}=A \omega^{s},
$$

where $\omega$ is the angular frequency, $A$ is a constant, $s \leq 1$ is the frequency exponent, $\sigma_{\text {tot }}$ is the measured total conductivity and $\sigma_{\mathrm{dc}}$ is the dc electrical conductivity.

Experimental results on the frequency dependence of the total measured conductivity $\sigma_{\text {tot }}(\omega)$ have been obtained. Figure 3 shows this dependence for as-deposited $\mathrm{Ag}_{33} \mathrm{Sb}_{31} \mathrm{Se}_{36}$ thin film sample having thickness $147.64 \mathrm{~nm}$ (as a representative example) at various fixed temperatures as $\ln \sigma_{\text {tot }}(\omega)$ versus $\ln \omega$. It is clear from Fig. 3 that $\sigma_{\text {tot }}(\omega)$ increases with frequency at any fixed temperature.

The frequency dependence of $\sigma_{\mathrm{ac}}(\omega)$ for $\mathrm{Ag}_{33} \mathrm{Sb}_{31} \mathrm{Se}_{36}$ thin films is represented in Fig. 4 as $\ln \sigma_{\text {ac }}(\omega)$ versus $\ln \omega$ for a thin film having thickness $180 \mathrm{~nm}$ at various fixed temperatures. It is clear from the figure that $\sigma_{\mathrm{ac}}(\omega)$ increases linearly with the frequency according to Eq. (1). Values of the frequency exponent $s$ have been calculated from the slopes of the straight lines of Fig. 4 and are plotted as a function of temperature in the inset of this figure. As can be seen, $s$ is smaller than unity and decreases with increasing temperature. The discrepancy in the value of $s$ at each temperature is indicated by vertical lines on the obtained results within the experimental error of $\pm 3 \%$. Accordingly, $s$ is independent of the film thickness in the investigated range.

According to the quantum mechanical tunneling (QMT) model [17], the exponent $s$ is almost equal to 0.8 and increases slightly with increasing temperature or is independent of temperature. Therefore, QMT model is considered not applicable to the investigated samples. 


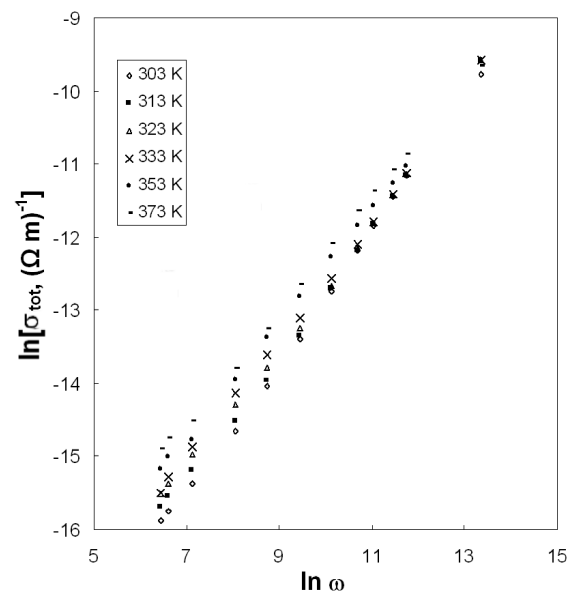

Fig. 3. The frequency dependence of the dc conductivity and the total measured conductivity $\sigma_{\text {tot }}(\omega)$ in $\mathrm{Ag}_{33} \mathrm{Sb}_{31} \mathrm{Se}_{36}$ thin film with thickness $147.64 \mathrm{~nm}$ at various fixed temperatures.

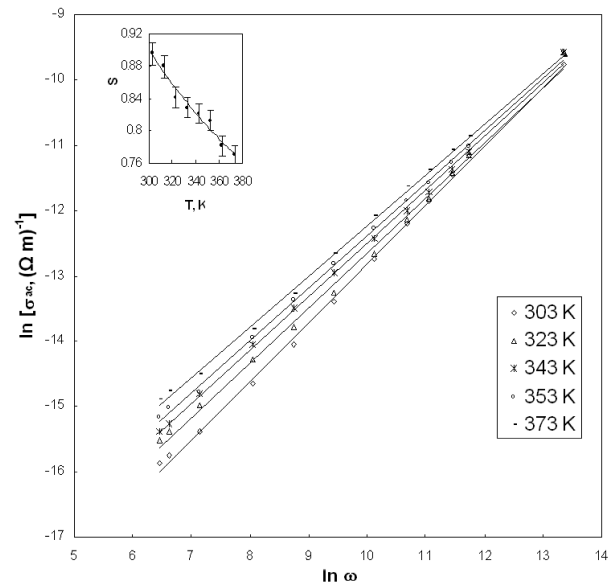

Fig. 4. Frequency dependence of $\sigma_{\mathrm{ac}}(\omega)$ for $\mathrm{Ag}_{33} \mathrm{Sb}_{31} \mathrm{Se}_{36}$ thin film (thickness $180 \mathrm{~nm}$ ) at various fixed temperatures; the inset shows the temperature dependence of the frequency exponent $s$ for this film.

According to the overlapping-large polaron tunnelling (OLPT) model [18], the exponent $s$ is both temperature and frequency dependent; $s$ decreases with increasing temperature from unity at room temperature to a minimum value at a certain temperature, and then it increases. Therefore, OLPT model is considered not applicable to the investigated samples.

In most of chalcogenide glasses, the obtained values of $s$ ranged from 0.7 to 1.0 at room temperature and have a tendency to decrease with increasing temperature [18]. Therefore the correlated barrier hopping ( $\mathrm{CBH}$ ) model [19] has 
been extensively applied to most of chalcogenide semiconductors. This is in good agreement with the obtained results, so the frequency dependence of $\sigma_{\mathrm{ac}}(\omega)$ can be explained in terms of CBH model. The expression for $s$ derived on the basis of this model proposed by Elliott $[19,20]$ can be written as

$$
s=1-6 k T / B,
$$

where $k$ is the Boltzmann constant, $T$ is the temperature in $\mathrm{K}$ and $B$ is the optical band gap of the material. Using the previously [16] obtained value of energy gap of $\mathrm{Ag}_{33} \mathrm{Sb}_{31} \mathrm{Se}_{36}$ thin films $(0.97 \mathrm{eV})$, the value of $s$ at room temperature is calculated using Eq. (2). The calculated value (0.838) is in good agreement with the experimental value (0.895) within $6.5 \%$.

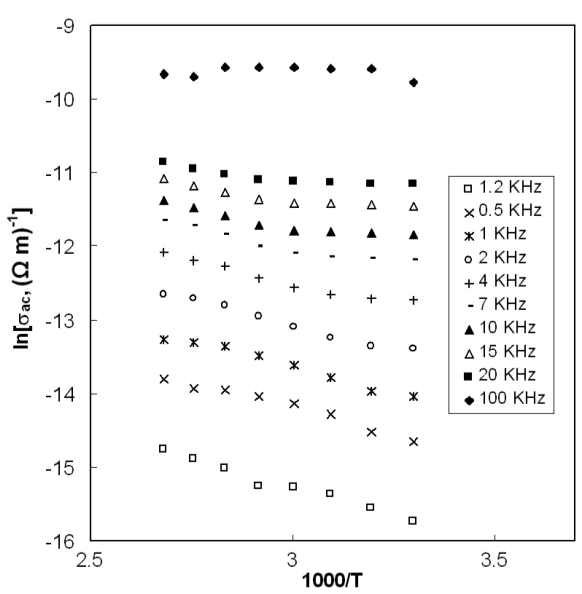

Fig. 5. Temperature dependence of the ac conductivity $\sigma_{\mathrm{ac}}(\omega)$ for $\mathrm{Ag}_{33} \mathrm{Sb}_{31} \mathrm{Se}_{36}$ thin film with thickness $180 \mathrm{~nm}$ at various frequencies.

The temperature dependence of $\sigma_{\mathrm{ac}}(\omega)$ for $\mathrm{Ag}_{33} \mathrm{Sb}_{31} \mathrm{Se}_{36}$ thin film of thickness $180 \mathrm{~nm}$ at different fixed frequencies is shown in Fig. 5 as $\ln \sigma_{\mathrm{ac}}(\omega)$ versus $10^{3} / T$ as a representative example. It is clear from this figure that $\sigma_{\mathrm{ac}}(\omega)$ increases nonlinearly with temperature especially at low frequency. A possible explanation of this behaviour may be that the density of states rises continuously toward the band edges instead of being sharp at a specific energy. The same behaviour for frequency and temperature dependence was obtained for films of different thicknesses in the investigated range.

\subsection{Frequency and temperature dependence \\ of dielectric constant $\varepsilon_{1}$ and dielectric loss $\varepsilon_{2}$}

The frequency and temperature dependence of $\varepsilon_{1}$ and $\varepsilon_{2}$ for $\mathrm{Ag}_{33} \mathrm{Sb}_{31} \mathrm{Se}_{36}$ thin films were studied in the range of temperature $303-373 \mathrm{~K}$ and frequency 0.1-100 kHz. The variation of $\varepsilon_{1}$ with the frequency for a film with thickness $160 \mathrm{~nm}$ is represented in Fig. 6 at various fixed temperatures. It is clear from 


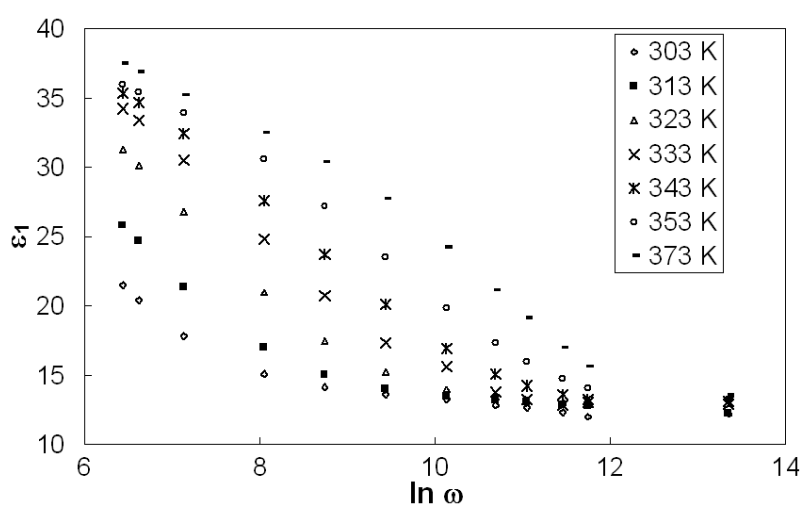

Fig. 6. Frequency dependence of the dielectric constant $\varepsilon_{1}$ for $\mathrm{Ag}_{33} \mathrm{Sb}_{31} \mathrm{Se}_{36}$ film of thickness $160 \mathrm{~nm}$ at various temperatures.

the figure that $\varepsilon_{1}$ is strongly frequency dependent at higher temperature, but its frequency dependence gets weaker as the temperature decreases. Also it is obvious that $\varepsilon_{1}$ increases with temperature and the increase is sharper at lower frequencies and higher temperature. The decrease in $\varepsilon_{1}$ with frequency can be attributed to the fact that, at low frequencies, $\varepsilon_{1}$ for polar material is due to the contribution of multicomponents of polarizability, deformational (electronic, ionic) and relaxational (orientational and interfacial). When the frequency is increased, the orientational polarization decreases since it takes more time than electronic and ionic polarization. This decreases the value of $\varepsilon_{1}$ with frequency reaching a nearly constant value at high frequency due to interfacial polarization. The increase in $\varepsilon_{1}$ with temperature is due to the fact that the orientational polarization is related to the thermal motion of molecules, because dipoles cannot orient themselves at low temperatures [21]. When the temperature is raised, the orientation of dipoles is facilitated and this increases the value of orientational polarization and consequently the value of $\varepsilon_{1}$. The dielectric polarization results from molecular dipoles $[22,23]$, which remain frozen at lower temperature $\left(T<T_{\mathrm{c}}\right)$ and attain rotational freedom at higher temperature $\left(T>T_{\mathrm{c}}\right)$ when the effect of the molecular interaction energy becomes weaker than that of the thermal energy [22]. Therefore, $\varepsilon_{1}$ increases with temperature.

Figure 7 shows the frequency dependence of $\varepsilon_{2}$ at different fixed temperatures for a film with thickness $160 \mathrm{~nm}$. It can be seen from the figure that $\varepsilon_{2}$ decreases with frequency and increases with temperature. The obtained data are plotted as $\ln \varepsilon_{2}$ versus $\ln \omega$ according to the equation [24]:

$$
\varepsilon_{2}=A \omega^{m}
$$

where $A$ is a constant. The power $m$ was calculated from the negative slopes of the obtained straight lines of Fig. 7 and represented as a function of temperature in the inset of this figure. It was found that $m$ decreases linearly with temperature according to Giuntini and Zancheha [25]: 


$$
m=-4 k T / W_{\mathrm{m}},
$$

where $W_{\mathrm{m}}$ is the maximum barrier height. The value of $W_{\mathrm{m}}$ was calculated according to Eq. (4) from the slope of the line in the inset of Fig. 7 and was found equal to $0.246 \mathrm{eV}$ which is in good agreement with the theory of hopping of charge carriers over a potential barrier as suggested by Elliott $[19,20]$ in case of chalco-

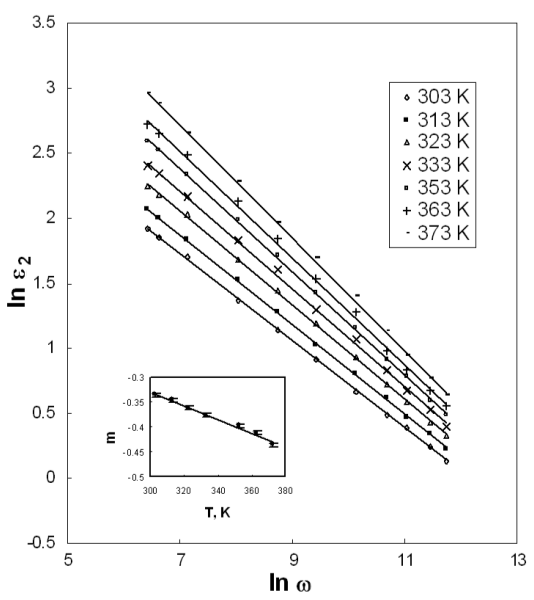

Fig. 7. Frequency dependence of the dielectric loss $\varepsilon_{2}$ for $\mathrm{Ag}_{33} \mathrm{Sb}_{31} \mathrm{Se}_{36}$ film (thickness $160 \mathrm{~nm}$ ) at various temperatures; the inset shows the temperature dependence of the parameter $m$ for this film.

genide glasses. The value of $W_{\mathrm{m}}$ is far smaller than $E_{\mathrm{g}}(0.97 \mathrm{eV})$ which reflects the influence of sample structure on the value of $s[26]$. Elliott further suggested that, in the case of single polaron, the energy required to remove an electron from a site to the infinity $\left(W_{\mathrm{m}}\right)$ is given by the relation $W_{\mathrm{m}}=$ (optical band gap) $/ 4$ $[19,27]$. The increase in $\varepsilon_{2}$ with temperature can be explained by Stevels [28] who divided the relaxation phenomena into three parts, conduction losses, dipole losses, and vibrational losses. At low temperature, conduction losses have minimum value which is proportional to $\sigma / \omega$. As the temperature increases, $\sigma$ increases and so the conduction losses increase which increases the value of $\varepsilon_{2}$ with temperature.

\subsection{Effect of annealing}

To illustrate the effect of annealing on the ac conductivity and dielectric properties of $\mathrm{Ag}_{33} \mathrm{Sb}_{31} \mathrm{Se}_{36}$ thin films, several films were annealed at 373 and $423 \mathrm{~K}$ for one hour. Using the same procedure as in Sects. 3.2 and 3.3, the frequency and temperature dependence of $\sigma_{\mathrm{ac}}(\omega), \varepsilon_{1}$, and $\varepsilon_{2}$ of the annealed films were also studied. Figure 8 shows the frequency dependence of $\sigma_{\mathrm{ac}}(\omega)$ at different elevated temperatures for $\mathrm{Ag}_{33} \mathrm{Sb}_{31} \mathrm{Se}_{36}$ thin film with thickness $160 \mathrm{~nm}$ and annealed at $423 \mathrm{~K}$ for $1 \mathrm{~h}$. It is clear from the figure that $\sigma_{\mathrm{ac}}(\omega)$ also increases linearly with the frequency according to Eq. (1). The same behaviour was obtained for all the 


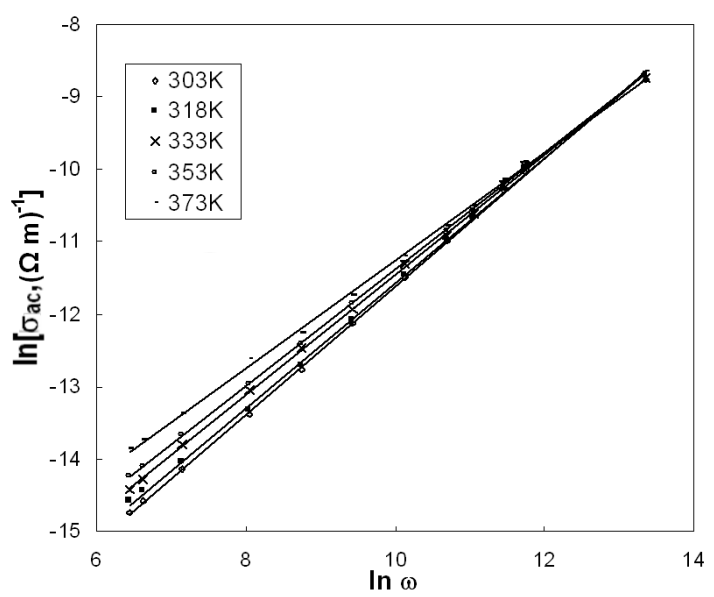

Fig. 8. Frequency dependence of $\sigma_{\mathrm{ac}}(\omega)$ for $\mathrm{Ag}_{33} \mathrm{Sb}_{31} \mathrm{Se}_{36}$ film having thickness $160 \mathrm{~nm}$ annealed at $423 \mathrm{~K}$ at different fixed temperatures.

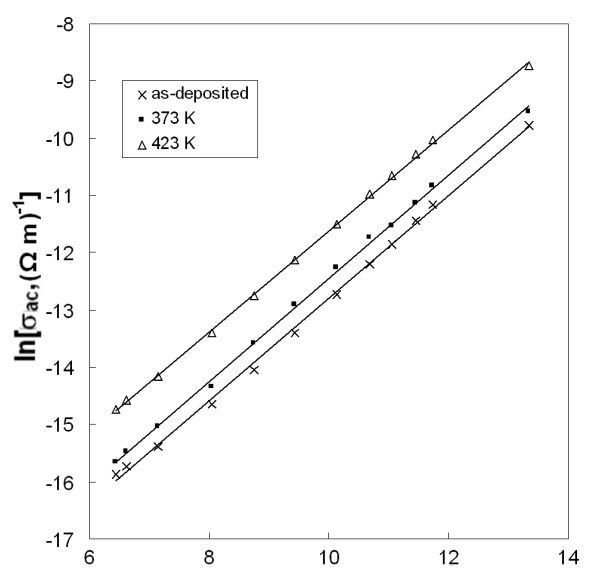

Fig. 9 $\ln \omega$

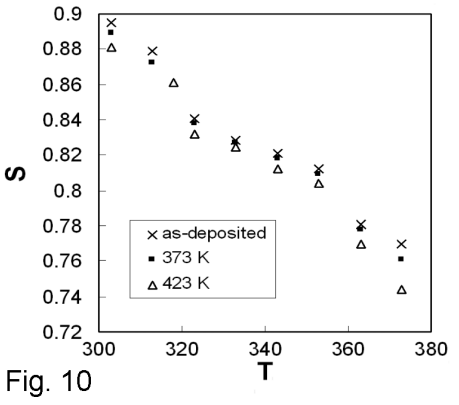

Fig. 10

Fig. 9. Frequency dependence of $\sigma_{\mathrm{ac}}(\omega)$ measured at room temperature for $\mathrm{Ag}_{33} \mathrm{Sb}_{31} \mathrm{Se}_{36}$ thin film (thickness $160 \mathrm{~nm}$ ) annealed at different temperatures.

Fig. 10. Temperature dependence of the frequency exponent $s$ for films annealed at different temperatures.

annealed films. The effect of annealing temperature on $\sigma_{\mathrm{ac}}(\omega)$ measured at room temperature at different fixed frequencies is represented in Fig. 9, from which it is obvious that $\sigma_{\mathrm{ac}}(\omega)$ increases with the annealing temperature. The frequency exponent $s$ was calculated as in Sect. 3.2 for all the annealed films and represented as a function of temperature in Fig. 10. It is clear from this figure that $s$ decreases with temperature which suggests that the frequency dependence of $\sigma_{\mathrm{ac}}(\omega)$ for the annealed films can also be explained by $\mathrm{CBH}$ model. Also, it is clear that at any temperature $s$ decreases with annealing temperature. The calculated values of 
$s$ according to Eq. (2) using our previous values of the energy gap [16] for the annealed films are also in good agreement with its experimental values.

The frequency and temperature dependence of the dielectric constant $\varepsilon_{1}$ and dielectric loss $\varepsilon_{2}$ were studied for the annealed samples as in Sect. 3.2. Figures 11 and 12 represent the frequency dependence of $\varepsilon_{1}$ and $\varepsilon_{2}$ measured at room temperature as an example for $\mathrm{Ag}_{33} \mathrm{Sb}_{31} \mathrm{Se}_{36}$ thin film of thickness $180 \mathrm{~nm}$ annealed at 373 and $423 \mathrm{~K}$ together with the as-deposited film. It is clear from these figures that both $\varepsilon_{1}$ and $\varepsilon_{2}$ increase with the increase in the annealing temperature. Values of $W_{\mathrm{m}}$ calculated from the frequency dependence of $\varepsilon_{2}$ as in Sect. 3.2 for the annealed films were found to be increased with the annealing temperature.

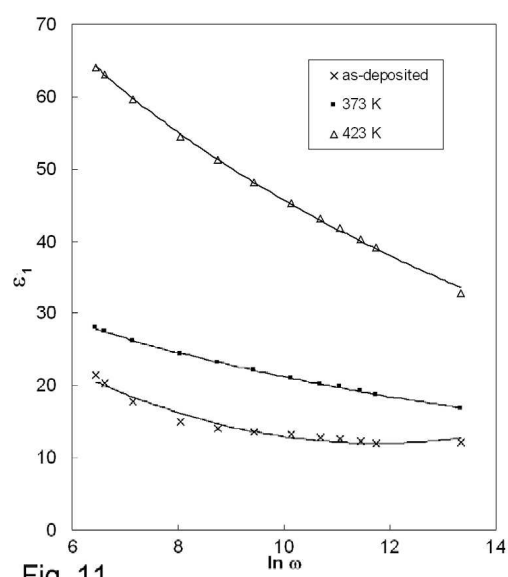

Fig. 11

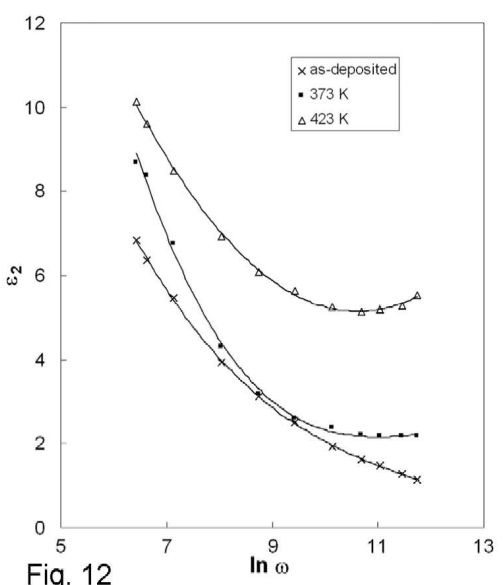

Fig. 12

Fig. 11. Frequency dependence of $\varepsilon_{1}$ measured at room temperature for $\mathrm{Ag}_{33} \mathrm{Sb}_{31} \mathrm{Se}_{36}$ thin film with thickness $180 \mathrm{~nm}$ and annealed at different temperatures.

Fig. 12. Frequency dependence of $\varepsilon_{2}$ measured at room temperature for $\mathrm{Ag}_{33} \mathrm{Sb}_{31} \mathrm{Se}_{36}$ film (thickness $180 \mathrm{~nm}$ ) and annealed at various temperatures.

The increase in $\sigma_{\mathrm{ac}}(\omega), \varepsilon_{1}$, and $\varepsilon_{2}$ with annealing temperature can be attributed to the change of the structure of the investigated films by annealing. Since the as-deposited amorphous thin films are characterized by the presence of high concentration of localized states in the band gap, during the process of annealing, the annealed out unsaturated bonds [29] produce a large number of saturated bonds. The reduction in the number of unsaturated defects decreases the density of localized states in the band structure and this increases the values of $\sigma_{\mathrm{ac}}(\omega)$, $\varepsilon_{1}$, and $\varepsilon_{2}$ for annealed films.

\section{Conclusions}

We have investigated for the first time the electronic conduction process in the $\mathrm{Ag}_{33} \mathrm{Sb}_{31} \mathrm{Se}_{36}$ system in the form of thin film samples, by measuring the 
frequency and temperature dependence of the ac conductivity and dielectric properties.

The ac conductivity $\sigma_{\text {ac }}(\omega)$ was found to be frequency and temperature dependent. The value of the frequency exponent $s$ and its temperature dependence confirmed the applicability of the $\mathrm{CBH}$ model to investigated thin film samples. The dielectric constant $\varepsilon_{1}$ and the dielectric loss $\varepsilon_{2}$ are frequency and temperature dependent. The dependence of $\varepsilon_{1}$ is attributed to interfacial and orientational polarization, respectively, while the temperature dependence of $\varepsilon_{2}$ is associated with the conduction loss.

Values of $\sigma_{\mathrm{ac}}(\omega), \varepsilon_{1}, \varepsilon_{2}$, and $W_{\mathrm{m}}$ were found to increase with the increase in the annealing temperature and attributed to the reduction in the number of unsaturated defects which decreases the density of localized states in the band structure.

\section{References}

[1] A.B. Seddon, J. Non-Cryst. Solids 184, 44 (1995).

[2] S.R. Elliott, Adv. Phys. 36, 135 (1987).

[3] E.H. Aly, A.M. Ibrahim, J. Phys. D 33, 2549 (2000).

[4] E. Abd El-Wahabb, M.M. El-Samanoudy, M. Fadel, Appl. Surf. Sci. 6766, 1 (2001).

[5] D. Tonchev, S.O. Kasop, J. Non. Cryst. Solids 248, 128 (1999).

[6] P.S. Nikam, H. S. Aher, Indian J. Pure Appl. Phys. 34, 393 (1996).

[7] H. Fritzsche, Philos. Mag. B 68, 561 (1993); A.V. Kolobov, S.R. Elliott, Adv. Phys. 40, 625 (1991).

[8] T. Wagner, P.J.S. Eween, J. Non-Cryst. Solids 266-269, 979 (2000).

[9] M. Ohto, M. Itoh, K. Tanaka, J. Appl. Phys. 77, 1034 (1995).

[10] K. Hirata, M. Kitao, S. Yamada, J. Phys. Soc. Jap. 52, 1317 (1983).

[11] M. Mitkova, Yu. Wang, P. Boolchand, Phys. Rev. Lett. 83, 3848 (1999).

[12] N. Nordman, O. Nordman, J. Appl. Phys. 90, 2206 (2001).

[13] M. Ohto, K. Tanaka, Appl. Phys. Lett. 71, 3409 (1997).

[14] H.A. Zayed, Thin Solid Films 274, 128 (1996).

[15] E. Abd El-Wahabb, Vacuum 57, 339 (2000).

[16] E. Abd El-Wahabb, E.A. Bekheet, Appl. Surf. Sci. 173, 103 (2001).

[17] A. Ghosh, Phys. Rev. B 41, 1479 (1990).

[18] A.R. Long, Adv. Phys. 31, 553 (1982).

[19] S.R. Elliott, Philos. Mag. B 36, 1291 (1977).

[20] S.R. Elliott, Adv. Phys. 36, 135 (1987).

[21] B. Tareev, Physics of Dielectric Materials, Moscow, Mir Publishers, 1979, p. 107.

[22] D.K. Goel, C.P. Singh, R.K. Shukla, A. Kumar, J. Mater. Sci. 35, 1017 (2000).

[23] K.K. Srivastava, D.R. Goyal, A. Kumar, K.N. Lakshminarayan, O.S. Panwar, I. Krishan, Phys. Status Solidi A 41, 323 (1977). 
[24] J.C. Giuntini, J.V. Zancheha, J. Non-Cryst. Solids 45, 57 (1981).

[25] J.C. Giuntini, J.V. Zancheha, J. Chem. Phys. 78, 383 (1981).

[26] A.A. Dokhel, Thin Solid Films 476, 366 (2005).

[27] J.A. Khan, C.A. Hogarth, J. Mater. Sci. 26, 17 (1991).

[28] J.M. Stevels, The Electrical Properties of Glass, Handbuch der Physik, Vol. 20, 1957, p. 350.

[29] S. Hasegawa, S. Yazalci, T. Shimizu, Solid State Commun. 26, 407 (1978). 